\title{
Unstable Angina in a Patient with Mucocutaneous Lymph Node Syndrome
}

\author{
Hideharu Hayashi, M.D., Kohsuke Kisamori, M.D., \\ Masanori Kaneko, M.D., Yoshinori Masumura, M.D., \\ Tadashi Kamikawa, M.D., Akira Kobayashi, M.D., \\ Yoshikazu Suzuki, M.D., Noboru Yamazaki, M.D., \\ Takashi Yamaguchi, M.D.,* and Yukio Harada, M.D.*
}

\section{SUMMARY}

An 11-year-old boy with mucocutaneous lymph node syndrome, who later developed an acute anteroseptal infarction and unstable angina, is presented. Anginal attacks were shown by 24hour long-term ambulatory electrocardiographic recording. He was shown to have multiple coronary aneurysms with thrombi, and underwent a successful aorto-coronary bypass.

\section{Additional Indexing Words:}

Coronary aneurysm Aorto-coronary bypass operation Beta thromboglobulin 24-hour long-term ambulatory electrocardiographic recording

$\mathbf{M}$ UCOCUTANEOUS lymph node syndrome (MCLS) ${ }^{1)}$ is a disease of unknown etiology that is most common in infants and children under 5 years of age. Although this disease has been known to have cardiovascular complications, angina is not a major presenting symptom.

We treated a boy who first presented with MCLS at the age of 11 years and who later at the age of 16 developed an acute anteroseptal infarction and unstable angina, as diagnosed by electrocardiogram and 24-hour long-term ambulatory electrocardiographic recording. He was shown to have coronary aneurysms with thrombi by coronary angiography and underwent a successful aorto-coronary bypass operation which resulted in resolution of his anginal symptoms.

From the Third Department of Internal Medicine, Hamamatsu University School of Medicine, Hamamatsu.

* The First Department of Surgery,

Address for reprint: Hideharu Hayashi, M.D., Third Department of Internal Medicine, Hamamatsu University School of Medicine, 3600 Handa-cho, Hamamatsu 431-31, Japan.

Received for publication March 2, 1983.

Manuscript revised September 5, 1983. 


\section{Case Report}

A 16-year-old boy had been in good health until 11 years of age when he developed typical signs of MCLS (Kawasaki disease), namely high fever for 9 days, conjunctivitis, lymphadenopathy, truncal skin rash, and erythema of both hands followed by desquamation of the fingertips. There were no overt cardiac abnormalities or electrocardiographic changes. At the age of 14 years, he started to experience frequent attacks of substernal chest pain on exertion.

On November 16, 1981, he developed severe chest pain when he was riding a bicycle. He was hospitalized for 2 months with a diagnosis of acute anteroseptal myocardial infarction (Fig. 1).

He was admitted to our hospital on January 28, 1982, as he subsequently noted frequent recurrent substernal chest pain both at rest and on walking. Initial physical examination revealed a late systolic click and late systolic Levine grade II murmur at the apex. The chest X-ray showed no cardiomegaly ( $\mathrm{CTR}=39 \%$ ); however, echocardiography revealed papillary muscle
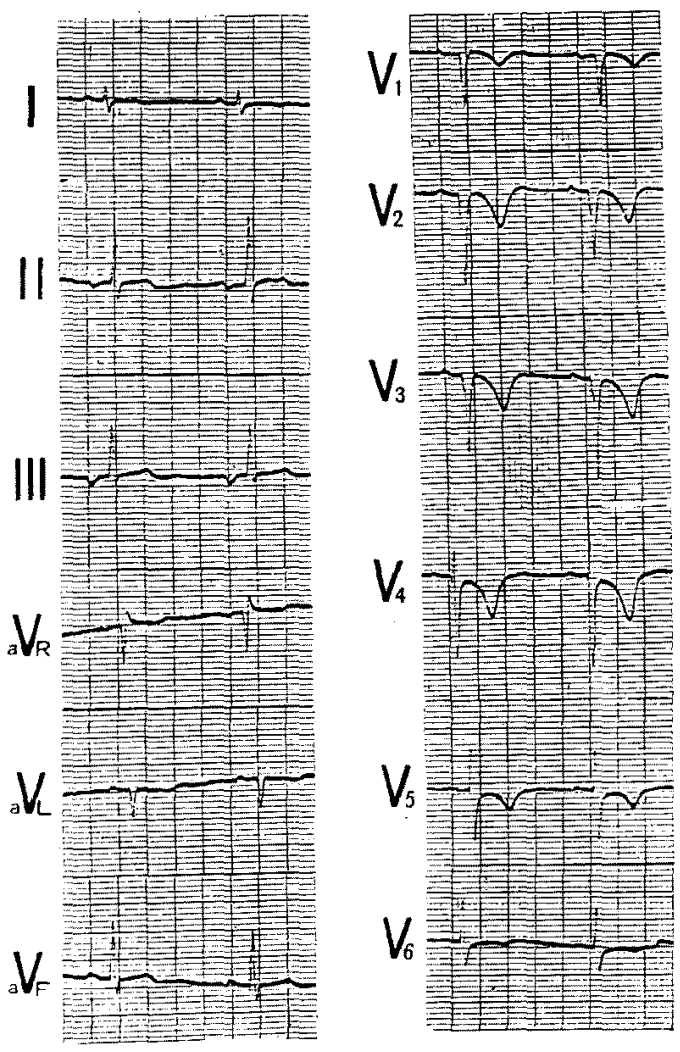

Fig. 1. Electrocardiogram at the time the patient was admitted to the hospital with a diagnosis of acute anteroseptal infarction. 
dysfunction of the posterior leaflet (Fig. 2).

The chest pain, which occurred at rest and on effort after he was admitted to our hospital, lasted 20 to $55 \mathrm{~min}$, and was not relieved by 0.3 to $0.6 \mathrm{mg}$ of sublingual nitroglycerin (Fig. 3). Twenty-four-hour long-term ambulatory electrocardiographic recordings were obtained. As shown in Fig. 4, the ST trend showed a prolonged period of ST segment depression, and the EGG revealed repeated ST segment depressions during this same $40 \mathrm{~min}$ period. Anginal attacks continued after combined administration of aspirin $330 \mathrm{mg}$, dipyridamole $150 \mathrm{mg}$ and nifedipine $30 \mathrm{mg} /$ day, but disappeared after increasing the dose of nifedipine to $60 \mathrm{mg} /$ day (Fig. 5). Levels of beta thromboglobulin, a platelet-specific protein, in platelet-poor plasma obtained during an anginal attack from peripheral venous samples were measured by radioimmunoassay. The level of beta thromboglobulin during an anginal attack was $20.1 \mathrm{ng} / \mathrm{ml}$, compared with levels of $8.0-11.0 \mathrm{ng} / \mathrm{ml}$ during angina free periods.

Coronary angiography and left ventriculographic studies were performed on February 10, 1982. The coronary angiography revealed three aneurysms of the left main coronary artery, the left anterior descending artery (LAD) and the right coronary artery (RCA). These aneurysms were apparently thrombosed, and the LAD and the RGA were occluded subtotally due to thrombi. The LAD was filled from collateral branches arising from the proximal RGA via the conus branch and the distal RCA was filled from collaterals from the left circumflex artery (LCX) (Fig. 6). Left ventriculography re-

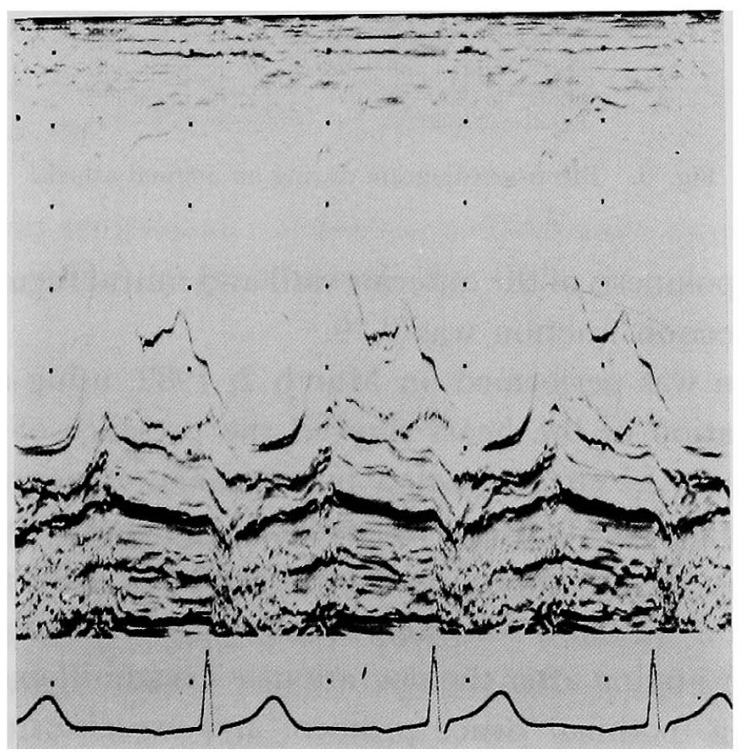

Fig. 2. Echocardiogram of the mitral valve, showing papillary muscle dysfunction of the posterior leaflet. 


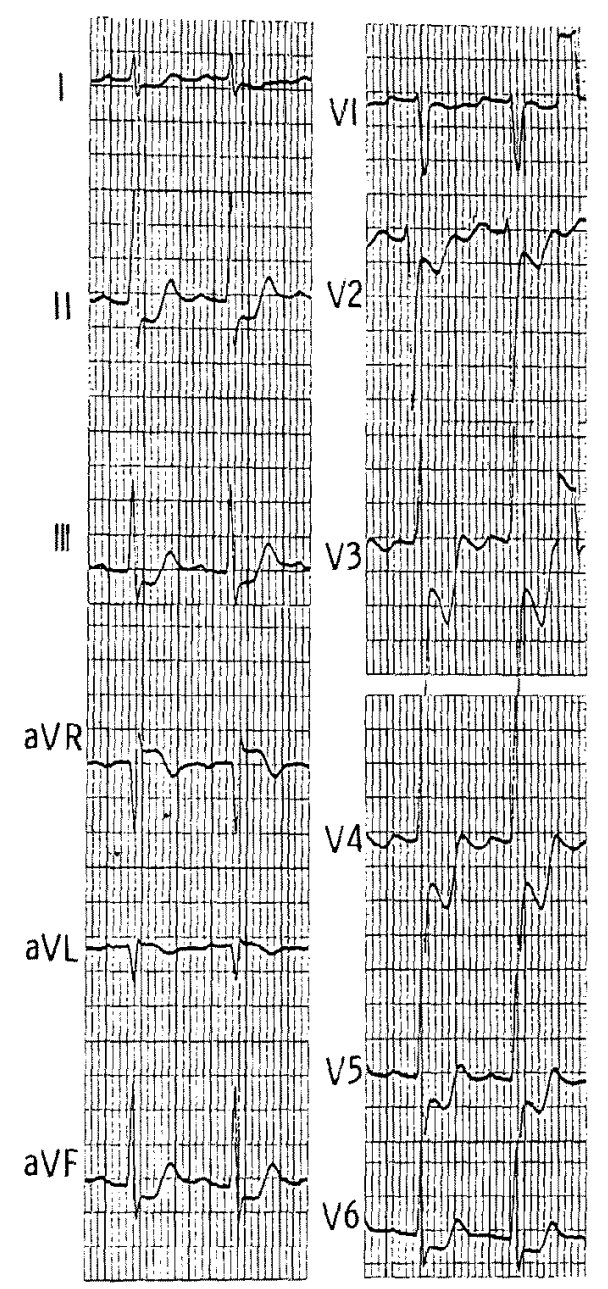

Fig. 3. Electrocardiogram during an anginal attack.

vealed a slight hypokinesis of the anterior wall and mitral regurgitation, Seller's grade I. The ejection fraction was 0.79 .

An operation was performed on March 2, 1982, using cardiopulmonary bypass. Examination of the heart showed the presence of clotted and calcified aneurysms at the sites revealed by coronary angiography. The saphenous veins were grafted between the ascending aorta and the LAD and the LCX, as the RCA was filled with collateral branches from the LCX.

The operation was well tolerated and the patient's recovery was rapid. He had no further angina after the operation. Treadmill exercise testing was performed using a modified Bruce protocol and exercise duration increased from $6.0 \mathrm{~min}$ (Bruce grade 1/2) to $12.5 \mathrm{~min}$ (Bruce grade 3) following the aorto-coronary bypass operation. On May 24, 1982, postoperative cardiac 
(1)

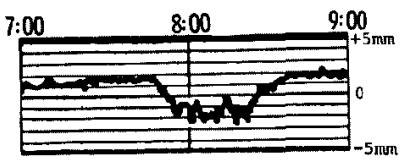

(2)

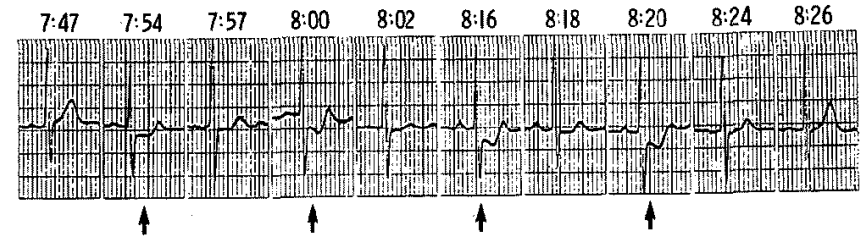

Fig. 4. (1) ST trend of 24-hour long-term ambulatory electrocardiographic recording. (2) Electrocardiogram $\left(\mathrm{CM}_{5}\right)$.

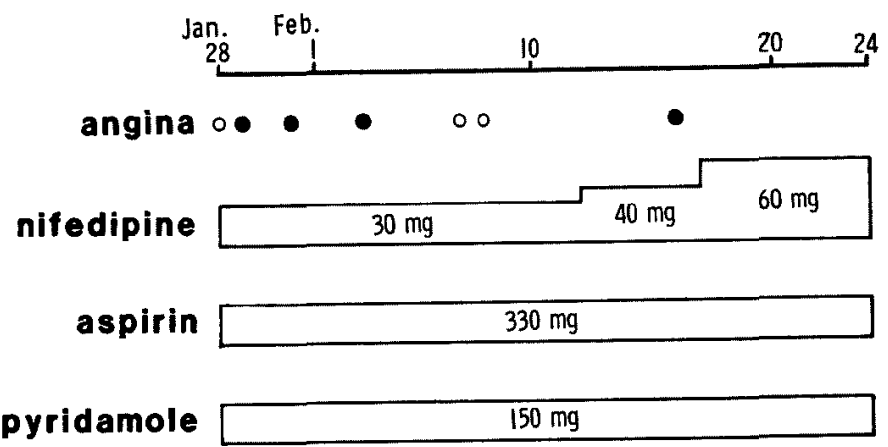

Fig. 5. Clinical course of the patient. $:$ angina lasting for longer than $20 \mathrm{~min}$. $O$ : angina of less than 20 min duration.

catheterization was performed. Coronary angiography demonstrated patency of the bypass graft with good runoff (Fig. 7).

\section{Discussion}

MCLS is a febrile disease most commonly seen in children in Japan which was first reported by Kawasaki in 1967.1) The clinical course of this disease had been thought to be benign until the appearance of reports of cardiovascular involvement such as myocarditis, ${ }^{2)}$ coronary aneuryms, ${ }^{3 /, 4)}$ myocardial infarction ${ }^{5)}$ and cardiac sudden death. ${ }^{6)}$ Angina ${ }^{7), 8)}$ is not a major presenting symptom of MCLS since MCLS is usually a disease of infants and young children who cannot adequately express symptoms such as chest pain.

The cardiac involvement ${ }^{9)}$ usually begins as a vasculitis of the microves- 

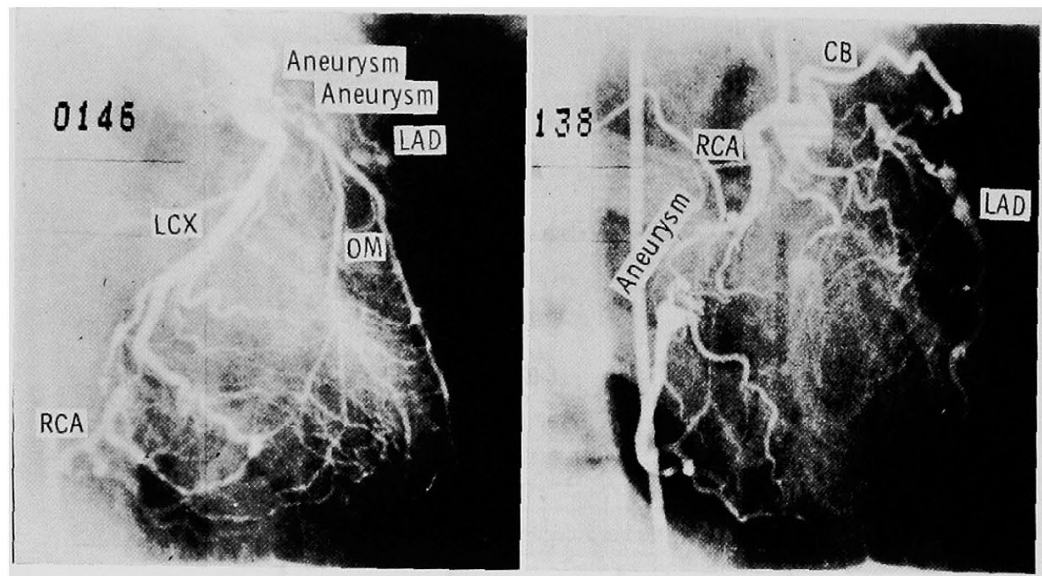

Fig. 6. Preoperative coronary angiography. Left photograph is a left coronary arteriogram in the right anterior oblique projection, and right photograph is a right coronary arteriogram in the right anterior oblique projection. $L A D=$ left anterior descending artery; $L C X=$ left circumflex artery; $\mathrm{OM}=$ obtuse marginal artery; $\mathrm{RCA}=$ right coronary artery; $\mathrm{CB}=$ conus branch.

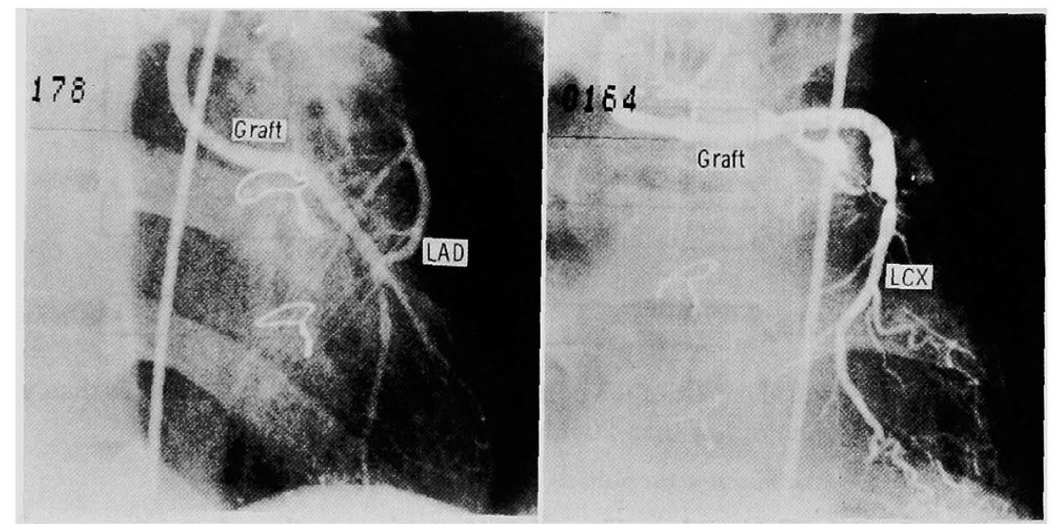

Fig. 7. Postoperative coronary angiography via saphenous vein graft. $\mathrm{LAD}=$ left anterior descending artery; $\mathrm{LCX}=$ left circumflex artery.

sels followed by a panvasculitis involving the three major coronary arteries. Aneurysms develop 12 to 25 days after the onset of the illness with possible subsequent intraluminal thrombosis and partial or total occlusion of the coronary vessels. After 1 month, the coronary inflammation usually resolves and the aneurysms generally disappear over the next 1 to 2 years, although they may persist as fibrotic or calcified aneurysms which may or may not thrombose and recanalize. In the case of large aneurysms or aneurysms with stenosis, the blood stream is converted to turbulent flow, ${ }^{10)}$ which may facilitate thrombus formation and platelet aggregation. ${ }^{11)}$ Fujiwara et al ${ }^{11)}$ reported that pathologic study showed coronary aneurysms due to angitis in 9 of the 15 
hearts from children with MCLS. At autopsy fresh large thrombi were seen in each aneurysm.

This patient developed chest pain on exertion at the age of 14 after he had first presented with characteristic manifestations of MCLS at the age of 11. At the age of 16 , he developed an acute anteroseptal infarction and frequent chest pain at rest and on effort. The attacks were severe and of long duration and were not relieved by nitroglycerin. The ST trend of a 24-hour long-term ambulatory electrocardiographic recording showed marked ST segment depression for a period of $40 \mathrm{~min}$. In this patient, anginal attacks were relieved by nifedipine, ${ }^{13), 14)}$ which is reported to be effective in vasospastic angina, but were not relieved by aspirin and dipyridamole, ${ }^{15)}$ which are inhibitors of platelet aggregation. Therefore, this patient's anginal attacks might have been caused by vasospasm. Elevation of beta thromboglobulin levels ${ }^{16), 17)}$ during angina may suggest enhanced platelet reactivity, and vasospasm might have been caused by thromboxane $A_{2}{ }^{18)}$ released by platelet thrombi that formed in the narrowed aneurysm.

This patient was thought to have unstable angina ${ }^{19)}$ and underwent coronary angiography which demonstrated coronary aneurysms with thrombi in the left main coronary artery, the LAD and the RGA. Unstable angina can be treated either or medically or surgically. ${ }^{20)-22}$ As his attacks were severe and he showed severely reduced exercise tolerance by treadmill test, we underwent aorto-coronary bypass operation. Patency of the graft was demonstrated by graft angiography, and improvement of exercise tolerance was shown by improvement of exercise duration on treadmill exercise testing.

\section{References}

1. Kawasaki T: Mucocutaneous lymph node syndrome: clinical observation in 50 cases. Jpn Allerg 16: 178, 1967 (in Japanese)

2. Onouchi Z, Tomizawa N, Goto M, Nakata K, Fukuda M, Goto M: Cardiac involvement and prognosis in acute mucocutaneous lymph node syndrome. Chest 68:297, 1975

3. Kawasaki T, Kosaki F, Okawa S, Shigematsu I, Yanagawa H: A new infantile acute febrile mucocutaneous lymph node syndrome (MLNS) prevailing in Japan. Pediatrics 54: 271, 1974

4. Kato H, Koike S, Yamamoto M, Ito Y, Yano E: Coronary aneurysms in infants and young children with acute febrile mucocutaneous lymph node syndrome. J Pediatr 86: 892, 1975

5. Yanagisawa M, Kobayashi N, Matsuya S: Myocardial infarction due to coronary thromboarteritis, following acute febrile mucocutaneous lymph node syndrome (MLNS) in an infant. Pediatrics 54: 277, 1974

6. Kegel SM, Dorsey TJ, Rowen M, Taylor WF: Cardiac death in mucocutaneous lymph node syndrome. Am J Cardiol 40: 282, 1977

7. Sandiford FM, Vargo TA, Shin JY, Pelargonio S, McNamara DG: Successful triple coronary artery bypass in a child with multiple coronary aneurysms due to Kawasaki's disease. J Thorac Cardiovasc Surg 79: 283, 1980 
8. Takeuchi Y, Suma K, Shiroma K, Asai T, Kusakawa S: Surgical experience with coronary arterial sequelae of Kawasaki disease in children. J Cardiovasc Surg 22: 231, 1981

9. Fujiwara H, Hamashima Y: Pathology of the heart in Kawasaki discase. Pediatrics 61: 100,1978

10. Yoshikawa T, Suma K, Sugawara M: Hemodynamic effects of bead-shaped aneurysm in Kawasaki's disease. Respiration and Circulation 28: 395, 1980 (in Japanese)

11. Haerem JW: Sudden coronary death: the occurrence of platelet aggregates in the epicardial arteries of man. Atherosclerosis 14: 417, 1971

12. Fujiwara H, Chen CH, Fujiwara T, Nishioka K, Kawai C, Hamashima Y: Clinicopathologic study of abnormal $Q$ waves in Kawasaki disease (mucocutaneous lymph node syndrome). Am J Cardiol 45: 797, 1980

13. Antman E, Muller J, Goldberg S, MacAlpin R, et al: Nifedipine therapy for coronaryartery spasm. New Engl J Med 302: 1269, 1980

14. Kimura E, Kishida H: Treatment of variant angina with drugs: a survey of 11 cardiology institutes in Japan. Circulation 63: 844, 1981

15. Danese CA, Haimov M: Inhibition of experimental artery thrombosis in dogs with plateletdeaggregating agents. Surgery 70:927, 1971

16. Sobel M, Salzman EW, Davies GC, Ch B, Handin RI, Sweeney J, Ploetz J, Kurland G: Circulating platelet products in unstable angina pectoris. Circulation 63: 300, 1981

17. Smitherman TC, Milam M, Woo J, Willerson JT, Frenkel EP: Elevated beta thromboglobulin in peripheral venous blood of patients with acute myocardial ischemia: direct evidence for enhanced platelet reactivity in vivo. Am J Cardiol 48: 395, 1981

18. Ellis EF, Oelz O, Roberts LJ II, Payne NA, Sweetman BJ, Nies AS, Oates JA: Coronary arterial smooth muscle contraction by a substance released from platelets: evidence that it is thromboxane $A_{3}$. Science 193: 1135, 1976

19. Russell RO, Resnekov L, Wolk M, Rosati $\mathrm{R}$, et al: Unstable angina pectoris: national cooperative study group to compare medical and surgical therapy. I. Report of protocol and patient population. Am J Cardiol 37: 896, 1976

20. Hulrgren HN, Pfeifer JF, Angell WW, Lipton MJ, Bilisoly J: Unstable angina: comparison of medical and surgical management. Am J Cardiol 39: 734, 1977

21. Russell RO, Resnekov L, Wolk M, Rosati R, et al: Unstable angina pectoris: national cooperative study group to compare medical and surgical therapy. II. In-hospital experience and initial follow-up results in patients with one, two and three vessel disease. Am J Cardiol 42: 839,1978

22. Cohn LH, Alpert J, Koster JK, Mee RBB, Collins JJ Jr: Changing indications for the surgical treatment of unstable angina. Arch Surg 113: 1312, 1978 